\title{
Current Status of Portable Insulin Infusion Devices
}

\author{
Satellite Workshop on Portable Insulin Infusion Devices. (Preceding the XVth Annual Meeting \\ of the European Society for Clinical Investigation) 18-19 March 1981, Basel, Switzerland
}

\author{
U. Keller ${ }^{1}$ and D. S. Schade ${ }^{2}$ \\ ${ }^{1}$ Department of Medicine, University Hospital, Basel, Switzerland and ${ }^{2}$ Department of Medicine, University of New Mexico, \\ Albuquerque, New Mexico, USA
}

On March 18 and 19, 1981, investigators and clinicians from Europe and the United States reviewed the current status of portable insulin delivery systems. Speakers representing 20 institutions and participants from 16 countries discussed technical and clinical aspects of this recently evolved alternative mode of insulin therapy in diabetic patients. The first day of the conference focused on the recent developments of portable infusion pump technology, supplemented by discussions of the advantages and disadvantages of current devices. On the second day, the available clinical long-term experience with insulin infusion devices was reported, and different views pro and con the various routes of insulin administration were presented.

\section{Current Insulin Pump Technology}

Detailed reports describing the current development of the Autosyringe, the Mill Hill, the Miles Laboratory, and the Siemens Company insulin pumps indicated that more sophisticated, usually externally worn infusers will soon be commercially available in the United States and Europe. However, the overall size of the devices will not be greatly reduced in the foreseeable future since current pump design requires space for the manual controls, batteries, electronics and insulin reservoir. Additional miniaturization of insulin pumps implies an increasing difficulty to deliver at precise rates. It also requires different technology, such as remote control programming and microelectronic circuitry. Prototypes of insulin infusion devices utilizing this miniaturization technology are currently being tested in the United States, but will not be generally available for the next few years. All participants at the meeting agreed that the most significant drawback to widespread clinical application of current insulin infusion pumps is the large size of the devices.

The problem of precipitation of regular insulin occurring in insulin reservoirs and delivery catheters was investigated by two groups. Insulin aggregation may be prevented in syringe-driven pumps if polyethylene catheters are used and if the insulin containing catheter and syringe are changed frequently. Furthermore, new insulin preparations will soon be marketed which have a decreased tendency for precipitation. Additives to currently-available insulin, such as polyethylene glycol or lysine, are effective in preventing precipitation but their toxicity in man is not well-defined, and they may interfere with the biological potency of insulin.

Concern for patient safety was a major topic discussed throughout the meeting. All pump manufacturers have now included alarms or safety devices to warn the patient of pump malfunction, and to provide an optical display, indicating proper operation. To date, serious consequences of pump failure have been minimal, but with increasing clinical use by patients with limited intellectual capabilities or inadequate training, more frequent and potentially serious accidents are anticipated. Data were presented indicating that rapid metabolic decompensation occurs within a few hours following accidental discontinuation of insulin supply since the patients lack a subcutaneous depot of insulin. An insulin flow monitor which activates an alarm if the pump stops or delivers an excess of insulin is the most desirable safety feature. Until such an alarm is available on all insulin delivery devices, clinical application of the pump should be restricted to patients who are knowledgeable about the use of their infusion device and who routinely perform home blood glucose monitoring to recognize early worsening of their metabolic control.

Although a reliable glucose sensor would be desirable, it is not yet available. Questions were raised concerning the need and function of such a device. If used directly to control the delivery of insulin from an insulin infusion pump, doubt was expressed that a glucose sensor could respond rapidly and appropriately to acute changes in plasma glucose, such as after meals, and malfunction of a glucose sensor might be extremely hazardous. However, a device which would non-invasively allow continuous blood glucose monitoring would be a major step forward.

\section{Routes of Insulin Delivery}

Proponents of the subcutaneous, intravenous, intramuscular, and intraperitoneal delivery routes presented their clinical data in insulin-dependent diabetic subjects. Although there is still disagreement as to the optimal route, it emerged from the discussions that there are unique advantages and disadvantages to each route. The subcutaneous insulin delivery route has been most extensively used in long-term studies since it has the principle advantage that it provides easy accessibility to a tissue with a large distribution area. A major disadvantage is the delayed absorption of insulin from the subcutaneous tissue, necessitating the initiation of a pre-prandial bolus dose of insulin at least $15 \mathrm{~min}$ before the meal. In addition, a subset of brittle diabetic patients has been identified which cannot be adequately treated by this delivery route. Some of these patients degrade insulin excessively in the subcutaneous tissue, and they can be successfully treated by the addition of a protease inhibitor to the insulin. However, other brittle insulin-dependent diabetic subjects do not respond to this form of treatment, and they require in- 
sulin administration by another route, usually the intramuscular or intravenous. Although the intramuscular route is readily accessible, technical problems with long-term usage have occurred, including muscle fibrosis, inadvertent catheter withdrawal, and catheter obstruction from blood clotting.

Two investigative groups reported on their successful use of prolonged intraperitoneal delivery to treat insulin-dependent diabetic patients. Several patients have now been treated for greater than three month's duration, one patient with an implanted device and remote control. The intraperitoneal delivery of insulin is characterized by rapid absorption of the hormone allowing administration of the pre-prandial dose immediately before the start of a meal. The route avoids peripheral hyperinsulinaemia, while glucose profiles are restored to normal. For implantable insulin delivery systems, this route has the advantage that the delivery catheter does not become obstructed by blood clots during prolonged use. However, experience with this route is limited and difficulty of access still restricts its widespread use.

Four investigators from Europe presented data of their experience with long-term intravenous insulin delivery by portable insulin infusion pumps. Near-normal glucose control can be achieved even in brittle diabetic patients with this route. Access to this delivery route is relatively invasive and difficult, since the catheter must be placed in a large central vessel via the subclavian vein. Whether the potential disadvantages of this route, such as thromboembolic complications, septicaemia, and limitation in patient activities, can be overcome, will determine the ultimate applicability of this route.

In summary, four insulin delivery routes have been examined for use by insulin infusion pumps, and specific benefits and drawbacks of each route defined. For the majority of patients, the subcutaneous access is preferred, but in brittle diabetic patients and in implantable infusion systems, other infusion routes are preferable.

\section{Clinical Application of Insulin Infusion Pumps}

Several years have now elapsed since the introduction of portable insulin infusion pumps by clinical investigators. The feasability of short-term open loop insulin therapy and the associated improvement in metabolic control has been reported previously, and the emphasis during the conference was placed on long-term experience and on patient's acceptance of current devices. The question of whether insulin infusion pumps should be used for clinical indications in certain insulin-dependent diabetic patients was raised. A spectrum of opinions was voiced from the statement that insulin infusion therapy should only be used experimentally to the opinion that treatment with portable insulin infusion devices may be appropriate for temporary use in pregnant diabetics, or for the calibration of insulin requirements in insulin-dependent diabetics. It was emphasised that long-term benefits from insulin pump therapy are not yet established, but an improvement of neuropathy, retinopathy and nephropathy was observed in several patients. In addition, other benefits related to the improvement in diabetic control were reported by several speakers. A reduction in hypoglycaemic reactions, and an improved sense of well-being was frequently noted. This benefit was described as a reduction in lethargy and in an improved mood which may also have been influenced by psychological factors related to the increased attention given to the patient by the physician. But the gain in freedom to arrange daily activities, such as timing and quantity of meals and exercise, was clearly pump-related. All participants agreed that if the patient is to benefit from insulin therapy, both patient and physician must be knowledgeable in the proper application of the device in order to obtain a benefit from the treatment. The patient should be willing to perform frequent home blood glucose monitoring in order to adjust the insulin dose if necessary.

It was pointed out that criteria to select appropriate patients have not been published by any major diabetic organization. Suggestions were made that the minimal criteria for portable insulin infusion therapy include only patients who 1) are not satisfactorily controlled by conventional therapy, and 2) can manage the pump themselves and respond adequately to its malfunction, and 3) have the possibility to consult permanently a physician who is familiar with the correct usage of insulin infusion devices. Whether externally worn or implantable systems will first achieve the status of standard therapy is entirely unknown at present. Furthermore, the question if insulin pump therapy delays the development of diabetic complications, remains to be determined in future clinical trials.

\section{Ulrich Keller M. D.}

Department of Medicine

Division of Endocrinology and Metabolism

University Hospital

CH-4031 Basel, Switzerland 